\title{
Analysis on the significance of optimizing the measurement standard management system in power industry
}

\author{
Yudan Liu ${ }^{1}$, Dan $\mathrm{Li}^{2}$, Yidan Zhang ${ }^{3}$, Wenyu Cheng ${ }^{4}, \mathrm{Ke} \mathrm{Wang}^{5}$ \\ ${ }^{1}$ State Grid Liaoning marketing service center, Shenyang,110003, China \\ ${ }^{2}$ State Grid Liaoning marketing service center, Shenyang,110003, China \\ ${ }^{3}$ State Grid Liaoning marketing service center, Shenyang,110003, China \\ ${ }^{4}$ State Grid Liaoning marketing service center, Shenyang,110003, China \\ ${ }^{5}$ State Grid Liaoning marketing service center, Shenyang,110003, China
}

\begin{abstract}
Measurement standard management is an important link to ensure the unity of national measurement unit system and the consistency and accuracy of value transfer. The establishment of measurement standard management system aims to provide technical support for traceability of social measurement. On the basis of ensuring the unity of national measurement unit system and the accuracy and reliability of measurement value, it undertakes the task of legal measurement. Its management level, technical ability and service quality not only directly determine the correctness of the measurement results, but also have a crucial impact on the social activities related to the measurement results. Based on the analysis of the current situation of measurement standard management in power industry, this paper expounds the significance of continuous optimization of measurement standard management system and the results achieved.
\end{abstract}

\section{INTRODUCTION}

In order to ensure the unity of national measurement unit system and the consistency and accuracy of measurement value transfer, and to provide fair and accurate verification and calibration data or results for national economic development, the State implements assessment system for some important measurement standards and includes them into the management scope of administrative permission. Measurement standard assessment is the assessment of the measurement ability of the national competent department and the confirmation of the qualification of carrying out the quantity value transmission by using the standard. The measurement standards to be assessed should not only meet the corresponding technical requirements, but also meet the relevant requirements of national legal management. Measurement standard assessment is not only a basic content of measurement supervision, but also an important technical basis for implementing the "measurement Law of the people's Republic of China" and ensuring the consistency of national measurement values.

Measurement standard management is an important part of the measurement work in the power industry. With the continuous expansion of the scale of the power industry, the number of all kinds of measurement standard devices is increasing year by year, the installation and use range is becoming wider, and the demand for traceability and transmission of measurement value is also growing. The traditional management method of measurement standard has been difficult to meet the current demand.

1 Current situation analysis

Measurement standard management has the features of long management cycle, huge contents and wide coverage. Under the new requirements in the new situation, there are still some problems in the management of measurement standards in the power industry, which need to be solved urgently.

First, it is difficult to supervise the measurement standard device. There are growing defects in the original management pattern. including the standard device is not submitted for inspection in time, the validity period of the verification certificate of measurement standard device is discontinuous, the management standard of measurement standard device is inconsistent, application for scrapping / deactivation is not in time, it is difficult to compare data,etc.

Second, the management of measurement standards is not centralized. The working mode that the user unit of the measurement standard device manages the information of

*Corresponding author's e-mail: lyd1_yf @ln.sgcc.com.cn 
the measurement standard device independently has weak linkage between the upper and lower levels, and the information integration is not good enough, which leads to the phenomenon of repeated submission of repeated data. The cycle of collecting data and reporting level by level is long, which leads to the poor timeliness of the information.

The third is the high frequency of changing measurement standard management personnel. It is difficult to solve the inherent problems such as insufficient proportion of professional technical personnel, lack of professionalism of personnel engaged in standard management posts or improper work handover.

\section{Significance of optimizing measurement standard management system}

2.1 optimizing the management system of measurement standards is the key link to ensure the accuracy and reliability of the verification results of measuring instruments in the power industry

Measurement standard management is an important link to ensure the unity of national measurement unit system and the consistency and accuracy of quantity value transmission. It provides fair and accurate verification and calibration data and results for national economic development and measurement supervision and management. The optimization of measurement standard management system aims to provide technical support for social measurement traceability. On the basis of ensuring the unity of national measurement unit system and the accuracy and reliability of measurement value, the management level, technical ability and service quality of measurement standard management system not only directly determine the correctness of measurement results, but also have a crucial impact on social activities related to measurement results.

2.2 optimizing the measurement standard management system is an important measure to improve the whole process management and control of measurement assets in the power industry

With the further deepening of the reform of the development concept of "safety, high quality, economy, eco-friendly and high efficiency", continuous optimization of measurement standard management in the power industry is a favorable catalyst to promote the measurement closer to the goal of "standardization, unification and informatization". At the same time, the overall operation concept of the society is gradually changing towards the open and sharing mode. The whole life asset management of the measurement standard device needs to be integrated with the operation concept in business, so as to promote the comprehensive level of measurement asset management. Through system interconnection, information sharing and other ways, the management of all aspects of asset life- cycle management of measurement standard device can be realized, and the management of visual monitoring can be realized, which can fundamentally solve the problems of nonstandard management and untimely inspection of measurement standard device in power supply companies at all levels.

\section{3 optimizing the measurement standard management system is an innovative breakthrough to actively improve the service quality and optimize the power business environment}

In order to thoroughly implement Socialism with Chinese Characteristics in the New Period of Xi Jinping and spirits of The 19th National Congress of CPC, we should actively implement the general requirements of the State Council on promoting the business environment construction, and always adhere to the customer centered and marketoriented service concept, constantly improve the comprehensive service level of enterprises, innovate the management system of measurement standards, establish and improve the management process of measurement standards, and streamline the plan. We should optimize the management mode of measurement standard, improve the quality and efficiency of measurement standard management, take the construction outline of ubiquitous power Internet of things as the focus, and achieve the management and control objectives of improving the construction of measurement standard system, organic integration of various businesses, and interactive sharing of relevant data.

\subsection{Optimizing the management system of measurement standards is the inevitable choice to serve the economic and social development}

Measurement standard management is an important link to protect power customers from economic losses caused by inaccurate measurement, and it is a strong support to supervise the accuracy and reliability of verification data of electric energy measuring instruments. The criterion of "justice, science, precision and efficiency" is not only the criterion of maintaining fairness and justice, but also the inevitable choice of serving economic and social development. Continuously optimize the concept of measurement standard management system, fully integrate the needs of economic and social development, combine with the construction outline of ubiquitous power Internet of things, continuously optimize management process, innovate management mode, improve management quality and efficiency, and achieve the management objectives of improving the construction of measurement standard system, organic integration of various businesses, and interactive sharing of all data. 

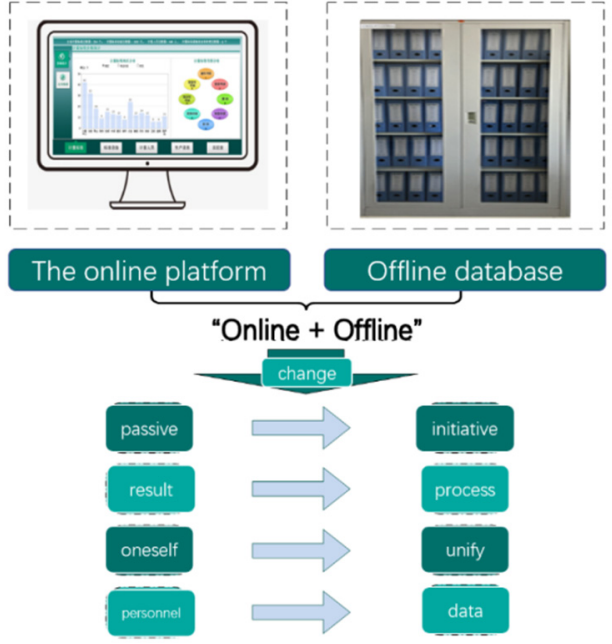

Figure 1 "online+offline"

\section{Conclusions}

\subsection{Three levels of management}

One is to change from "result control" to "process control". In order to solve the problem that the supervision of measurement standard device is difficult, the online measurement system management platform and offline provincial standard device database are established. Through the "Online + offline" two pronged management mode, the management mode of "result oriented, problemsolving again" is realized to "strictly grasp the whole process of management, find problems at any time and solve them at any time".

The second is to change from "independent management" to "unified management and control". In order to solve the problem that the information management of measurement standard is not centralized, the working idea is changed rapidly. The design concept of multi-layer nesting and folding expansion is used to establish the database of measurement standard device. The data information of measurement standard device is transferred from the local company independent management to the provincial unified management. It can realize the whole process management and control, reduce the invalid submission, eliminate the phenomenon of "annual inspection and annual submitting data", and simplify the work-flow. At the same time, the leading experts in the industry are widely absorbed, and the measurement standard management expert group is established. Aiming at the problems in the measurement standard management process of the power industry, "timely pulse and joint diagnosis" is realized, and the management mode of "zero time difference and zero precipitation" is realized to provide professional support for the measurement standard management of grass-roots units.

Third, from "control personnel" to "control data".In order to solve the problem of frequent replacement of measurement standard management personnel, innovate the management mode, and gradually change the management mode from focusing on the management and control of management personnel to the management and control of data. Through the development of practical, comprehensive and specific management system, the information collection template and filling instructions of measurement standard device are issued, the measurement standard information is collected, and the common problems of measurement standard management are compiled. In order to update the information in the database, realize the real-time control of the information of the measurement standard device of the local and municipal companies, absorb the leading experts to set up the measurement standard management expert group to answer the management problems in real time, so as to unify and centralize the management and control of the provincial standard device, so as to make the management and control of the standard device institutionalized, programmed, real-time and whole process, even if the management personnel is replaced, minimizing the risk and information loss caused by improper handover.

\subsection{Two improvements in economic benefits}

First, achieving accurate measurement and continuous focus. With the steady development of the power industry and the increasing awareness of the people to protect their rights, the accurate and reliable measurement results of electric energy measuring instruments have become the key link to contact power enterprises and power customers. In order to protect the rights and interests of power enterprises and customers from loss, it is very important to strictly observe the quality control of measurement standard device. The whole process management and control of grid measurement standard management aims to strengthen the supervision of measurement standard devices, continuously improve the professional technical ability and service level, ensure the accuracy and reliability of the verification results of electric energy measuring instruments from the source of verification, and contribute to the continuous focus of power enterprises on serving the local economic development.

Second, strengthen management and control, reducing costs and increasing efficiency. With the continuous upgrading of electric energy measuring instruments, the verification capacity of some old measuring devices cannot match the current verification requirements. Continuous optimization of measurement standard management system can track the demand of power enterprises for devices and the use of measurement standard devices at any time, reasonably allocate verification resources, reduce the verification cost of idle measurement standard devices and maintenance costs of old measurement standard devices, effectively promoting the measurement standard devices, both the number and the device quality can be strictly controlled, to further improving the management and control level of measurement management.

\section{Reference:}

[1] JJF1033《Standards of measurement and assessment》, National Technical Committee of Measurement by 
Legal System Administration

[2] JJF1069 《Standards for the assessment of legal metrological verification institutions》, Metrology Department of general Administration of Quality Supervision, Inspection and Quarantine

[3] Analysis of relevant measures for applying for metrological standard assessment by legal metrological technical institutions at the grassroots level, Bin Guan,

[4] A brief discussion on the application, acceptance and evaluation procedure of measurement standard assessment, Shan Ling

[5] Interpretation of 8 preparatory works for the assessment of new metrological standards for application, Limin Sun 